\title{
Treatment of localized neuropathic pain after disk herniation with $5 \%$ lidocaine medicated plaster
}

This article was published in the following Dove Press journal:

International Journal of General Medicine

16 August 2012

Number of times this article has been viewed

Rudolf Likar'

Ingo Kager'

Michael Obmann'

Wolfgang Pipam'

Reinhard Sittl ${ }^{2}$

'Department of Anesthesiology and Intensive Care, Klagenfurt Hospital, Klagenfurt, Austria; ${ }^{2}$ Department of Anesthesiology, Interdisciplinary Pain Center, University Hospital Erlangen, Erlangen, Germany
Correspondence: Rudolf Likar Department of Anesthesiology and Intensive Care, Landeskrankenhaus Klagenfurt, St Veither Strasse 47, 9026 Klagenfurt, Austria

Tel +4346353826120

Fax +4346353823070

Email rudolf.likar@kabeg.at
Objective: To assess treatment with the 5\% lidocaine medicated plaster for peripheral neuropathic pain after disk herniation.

Study design: Case series, single center, retrospective data.

Patients and methods: Data of 23 patients treated for neuropathic pain with the lidocaine plaster for up to 24 months after a protrusion or prolapse of the cervical, thoracic, or lumbar vertebral disks were retrospectively analyzed. Changes in overall pain intensity, in intensity of different pain qualities and of allodynia and hyperalgesia were evaluated.

Results: Patients (14 female/nine male, mean age $53.5 \pm 10.4$ years) presented with radiating pain into the abdomen, back, neck, shoulder, or legs and feet with a mean pain intensity of $8.3 \pm 1.5$ on the 11-point Likert scale. Mean treatment duration was 7.6 months; $52 \%$ of the patients received lidocaine plaster as monotherapy. At the end of the observation, mean overall pain intensity had been reduced to $3.1 \pm 1.8$. All other parameters also improved. The treatment was well tolerated.

Conclusion: These results point to a safe and effective treatment approach with $5 \%$ lidocaine medicated plaster for localized neuropathic pain related to disk herniation. However, owing to the small sample size, further investigation in a larger-scale controlled trial is warranted.

Keywords: peripheral neuropathic pain, radiating pain, topical analgesic

\section{Introduction}

Neuropathic pain has recently been redefined as "pain arising as a direct consequence of a lesion or disease affecting the somatosensory system." ${ }_{1,2}$ It can be of peripheral or central origin depending on the location of the lesion or disease within the nervous system, with distinct differences regarding underlying pathophysiology, clinical manifestations, and treatment requirements. ${ }^{3}$ Neuropathic pain affects up to $8 \%$ of the general European population ${ }^{4}$ and is considered under-recognized and often undertreated..$^{5}$ Health-related quality of life is often markedly impaired ${ }^{5,6}$ and the economic burden is substantial. ${ }^{5}$

The topical analgesic 5\% lidocaine medicated plaster (Versatis ${ }^{\circledR}$, Grünenthal $\mathrm{GmbH}$, Aachen, Germany) has been shown efficacious and safe in relieving neuropathic pain in pain syndromes such as postherpetic neuralgia 7,8 and diabetic polyneuropathy, ${ }^{8}$ and is recommended as first-line treatment for localized, peripheral neuropathic pain. ${ }^{9}$ Beneficial effects have also been observed in other primarily neuropathic conditions such as painful idiopathic sensory polyneuropathy, complex regional pain syndrome, carpal tunnel syndrome sequelae, and postsurgical and posttraumatic pain or mixed pain conditions such as low back pain; ${ }^{10}$ however, earlier low 
back pain studies have lacked a clear diagnosis concerning the origin of pain and their findings therefore need to be interpreted with caution. ${ }^{10}$

Pain relief using a 5\% lidocaine medicated plaster at the site of pain is achieved by a dual mode of action: the plaster itself provides the perception of immediate cooling and mechanical protection and its active compound lidocaine acts pharmacologically as a voltage-gated sodium channel inhibitor, stabilizing the neuronal membrane potential on abnormally excited A-delta and C fibers. ${ }^{10}$ This latter mode of action of lidocaine is still not fully understood and is being discussed in the scientific community.

Disk herniation is the most common cause of radicular pain; inflammation of the affected nerve as well as compression of a dorsal root ganglion all appear to play a part in generating pain. ${ }^{11}$ As the $5 \%$ lidocaine medicated plaster has proven beneficial in other neuropathic pain conditions, ${ }^{10}$ the authors considered it might also provide relief for peripheral radiating neuropathic pain after disk herniation. We therefore retrospectively analyzed data on the use of lidocaine plaster in the treatment of this type of pain.

\section{Methods}

This study was a retrospective analysis of all patients treated for radiating neuropathic pain following a protrusion or prolapse of the cervical, thoracic, or lumbar vertebral disks with the 5\% lidocaine medicated plaster at the Center for Interdisciplinary Pain Therapy and Palliative Medicine in Klagenfurt, Austria. Only patients with a diagnosis of disk herniation with radiating neuropathic pain were included; patients with neuropathic pain resulting from other causes for example, postsurgical/scar pain - were excluded from the analysis. The use of up to three plasters was permitted for a maximum of 12 hours, with plaster-free intervals of at least 12 hours. Patients had been assessed at monthly intervals for a period of 6 months then followed up for up to 24 months. Data had been collected by the attending clinicians using standardized forms and included basic demographic information, lidocaine plaster treatment details, concomitant medications, pain intensity, presence/absence of allodynia, hyperalgesia and pain qualities such as burning pain, and the occurrence of adverse events. All pain parameters were rated on an 11-point Likert scale $(0=$ not present, $10=$ worst possible state; eg, worst imaginable pain for the pain-intensity parameter).

Data were retrieved from the center's database and retrospectively analyzed by one researcher; this analysis was approved by the center's ethics committee. Data were described as mean \pm standard deviation or number of patients. All data are "as observed"; missing data were not imputed.

\section{Results}

A total of 23 patients (14 female/nine male) were included in this analysis. Their mean age was $53.5 \pm 10.4$ years. Eight patients suffered from comorbidities (diabetes, hypertension [two patients], Still's disease, cancer [two], status post-carpal tunnel syndrome, status post-chest surgery). All patients suffered from neuropathic pain after a protrusion or prolapse of the cervical, thoracic, or lumbar vertebral disks. The pain was localized, radiating into the abdominal region (one patient), neck (three), shoulder (three), back (seven), or legs and feet (nine). Two patients had a medical history of laminectomy, however, their radiating neuropathic pain was considered unrelated to surgery. Mean pain intensity at baseline was $8.3 \pm 1.5$ on the 11-point Likert scale and mean pain duration was $9.13 \pm 7.84$ years. Five patients $(21.7 \%)$ presented with allodynia and seven patients $(30.4 \%)$ had hyperalgesia. The majority of patients suffered from burning pain (69.6\%) and neuralgic aching/radiating pain (91.3\%).

The 5\% lidocaine medicated plaster was administered as monotherapy to twelve patients $(52.2 \%)$ and as add-on to pre-existing pain medication to eleven patients (47.8\%), eight of whom used one additional analgesic and the other three used more than one. The additional analgesics included gabapentin (one patient), tricyclic antidepressants (two patients; amitriptyline), nonsteroidal anti-inflammatory drugs (five patients; diclofenac, naproxen, mefenamic acid), and opioids (five patients; tramadol, transdermal buprenorphine, oxycodone, hydromorphone, morphine hydrochloride). Mean duration of treatment with the 5\% lidocaine medicated plaster was 7.6 months (range 1-24 months). One patient discontinued plaster treatment after 1 month and a second discontinued after 2 months. The remaining 21 patients $(91.3 \%)$ completed the 6-month observation period and eight patients (34.8\%) administered the plaster for 12 or more months. The majority of the patients $(78.3 \%)$ administered only one plaster per day and the remaining five patients $(21.7 \%)$ used two plasters. The plasters were applied to the painful region - that is, to the neck, shoulder, back, or legs/feet.

Overall pain intensity and intensity of the two most prominent pain qualities, burning pain and neuralgic aching/ radiating pain, markedly decreased following treatment with 5\% lidocaine medicated plaster and remained at a low level throughout the observation period with final values of $3.1 \pm 1.8$ for overall pain intensity, $2.0 \pm 2.0$ for burning pain, 
and 3.0 \pm 1.7 for neuralgic aching/radiating pain at the end of observation (Figure 1). Improvement was also observed in the other parameters evaluated in this study: tingling sensation (pins and needles), from $1.0 \pm 2.7$ at baseline to $0.5 \pm 1.3$ at the end of observation; abnormal temperature sensation, from $1.1 \pm 2.7$ to $0.6 \pm 1.4$; paresthesias, from $1.5 \pm 2.9$ to $0.2 \pm 0.7$; hyperalgesia, from $2.4 \pm 3.8$ to $0.6 \pm 1.3$; and allodynia, from $1.8 \pm 3.5$ to $0.6 \pm 1.3$.

All 23 patients experienced marked reductions in pain intensity, independent of whether they presented with allodynia at baseline or not and including the two patients who discontinued the observation after 1 and 2 months, respectively. Allodynia markedly improved in the five patients presenting with this symptom at baseline but was still present after 6 months of treatment, though at a much lower intensity.

Administration of the $5 \%$ lidocaine medicated plaster was associated with five adverse events over the 6-month period. One case each of constipation, morning nausea, mild tachycardia, and mild stomach pains were reported in the first treatment month and one case of flatulence occurred in the second month; all adverse events were assessed as not being causally related to lidocaine plaster treatment.

\section{Discussion}

This retrospective analysis showed that administration of the 5\% lidocaine medicated plaster led to a marked reduction in pain intensity and all other evaluated pain parameters in patients with disk herniation suffering primarily from burning and neuralgic aching/radiating pain. It should be noted that these patients were diagnosed with neuropathic pain following disk herniation; their pain did not arise because of surgical interventions or other indications. The treatment was well tolerated over a mean treatment period of 7.6 months. Most patients (78\%) used only one plaster per day.

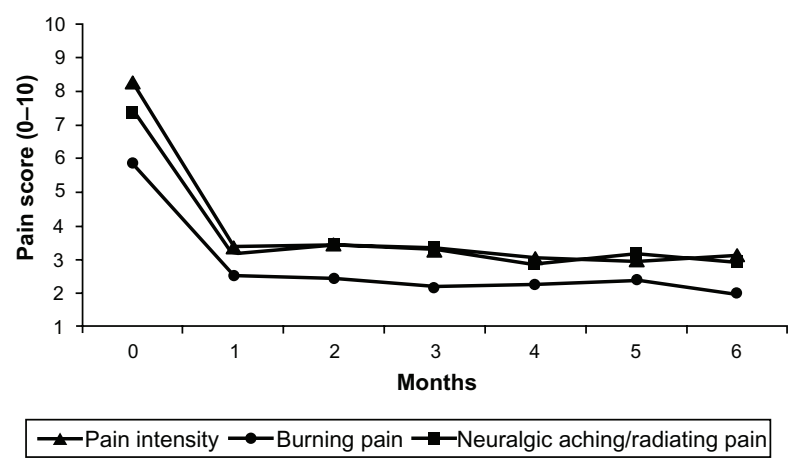

Figure I Mean overall pain intensity, burning pain, and neuralgic aching/radiating pain following treatment with $5 \%$ lidocaine medicated plaster in patients with disk herniation.

Notes: $\mathrm{n}=23$ at baseline, $\mathrm{n}=22$ at I month, $\mathrm{n}=2 \mathrm{I}$ at 6 months.
Approximately $30 \%$ of our patients suffered from back pain, whereas pain occurred in distant body parts in the remaining $70 \%$ (mainly lower extremities). It is likely that both nociceptive and neuropathic pain-generating mechanisms are involved in pain experienced by patients suffering from disk herniation. The pain is not due to a discharge exclusively in nociceptive afferents; rather, it is due to a heterospecific discharge in the affected nerve. ${ }^{11}$ Inflammation of the affected nerve, compression of a dorsal root ganglion, and, potentially, referred pain (through noxious stimulation of nerve endings within spinal structures such as disks, with pain spreading into the lower limbs) appear to play a part in generating pain. ${ }^{11}$

To our knowledge, there are only a few case reports describing the treatment of neuropathic pain with 5\% lidocaine medicated plaster after disk herniation. Most of the relevant literature - for example, studies on low back pain - does not include detailed primary diagnoses of the patients. A positive response to treatment was reported in a small case series of four patients suffering from low back pain. ${ }^{12}$ The combination of lidocaine plaster and gabapentin relieved burning pain in one patient with a herniated disk (L4-L5), and the addition of the plaster to the pain treatment regimen of one patient with a bulging disk (no frank herniation) also "helped about 80\%." In a recent larger case series including patients with chronic low back pain with neuropathic components, $76 \%$ of the patients were judged much or very much improved following treatment with 5\% lidocaine medicated plaster as determined by the Clinical Global Impression of Change. ${ }^{13}$ From the primary diagnosis of these cases we could only conclude for one patient without doubt that the neuropathic low back pain was related to disk herniation (discopathy). This patient showed much improvement under lidocaine plaster treatment.

The presence of allodynia has been shown to be a positive predictor for treatment success with the $5 \%$ lidocaine medicated plaster in neuropathic pain patients with various etiologies. ${ }^{14}$ However, in the present patient population, overall pain intensity was reduced regardless of whether patients presented with allodynia at the start of treatment. This finding is in agreement with Nicolaou et al's study ${ }^{13}$ in which it was observed that low back pain case reports did not show a clear association between the presence of allodynia and a positive treatment outcome, and patients without allodynia responded equally to treatment with the $5 \%$ lidocaine medicated plaster. Further studies are warranted to investigate the relationship between the presence of allodynia and treatment outcome and to assess allodynia outcome itself under treatment with $5 \%$ lidocaine medicated plaster. 


\section{Conclusion}

Although the number of patients evaluated in this study was small, the results are promising and point to a safe and effective treatment approach for the pain related to this disabling localized neuropathic pain condition. Further larger-scale and well-controlled trials investigating the use of the $5 \%$ lidocaine medicated plaster for peripheral neuropathic pain following disk herniation are warranted.

\section{Acknowledgments}

Special thanks go to study nurse Susanne Demschar. The authors also wish to thank E Grosselindemann (Brett Medical Writing, Bibra Lake, Australia) and B Brett (Brett Medical Writing, Pulheim, Germany) for writing and editorial assistance. All costs associated with the publication of the manuscript were met by Grünenthal GmbH, Aachen, Germany.

\section{Disclosure}

$\mathrm{RL}$ received lecture fees and honoraria for serving on advisory boards of Grünenthal GmbH, Aachen, Germany. RS received lecture fees from Grünenthal $\mathrm{GmbH}$, Aachen, Germany. MO, WP, and IK have no conflicts of interest to declare in this work.

\section{References}

1. Treede RD, Jensen TS, Campbell JN, et al. Neuropathic pain: redefinition and a grading system for clinical and research purposes. Neurology. 2008;70:1630-1635.

2. International Association for the Study of Pain [web page on the internet]. IASP Taxonomy. International Association for the Study of Pain; 2012 [updated May 22, 2012]. Available from: http://www.iasp-pain.org/AM/ Template.cfm?Section=Pain_Definitions\&Template=/CM/HTMLDisplay.cfm\&ContentID=1728. Accessed January 9, 2012.
3. Haanpää M, Backonja MM, Bennett M, et al. Assessment of neuropathic pain in primary care. Am J Med. 2009;122(10 Suppl):S13-S21.

4. Smith BH, Torrance N. Epidemiology of neuropathic pain. Pain Manage. 2011;1:87-96.

5. O'Connor AB. Neuropathic pain: quality-of-life impact, costs and cost effectiveness of therapy. Pharmacoeconomics. 2009;27(2):95-112.

6. Jensen MP, Chodroff MJ, Dworkin RH. The impact of neuropathic pain on health-related quality of life. Review and implications. Neurology. 2007;68(15):1178-1182.

7. Binder A, Bruxelle J, Rogers P, Hans G, Bösl I, Baron R. Topical 5\% lidocaine (lignocaine) medicated plaster treatment for post-herpetic neuralgia: results of a double-blind, placebo-controlled, multinational efficacy and safety trial. Clin Drug Investig. 2009;29(6):393-408.

8. Baron R, Mayoral V, Leijon G, Binder A, Steigerwald I, Serpell M. $5 \%$ lidocaine medicated plaster versus pregabalin in post-herpetic neuralgia and diabetic polyneuropathy: an open-label, non-inferiority two-stage RCT study. Curr Med Res Opin. 2009;25(7):1663-1676.

9. Dworkin RH, O'Connor AB, Audette J, et al. Recommendations for the pharmacological management of neuropathic pain: an overview and literature update. Mayo Clin Proc. 2010;85(3 Suppl):S3-S14.

10. Mick G, Correa-Illanes G. Topical pain management with the $5 \%$ lidocaine medicated plaster - a review. Curr Med Res Opin. 2012;28(6): 937-951.

11. Bogduk N. On the definitions and physiology of back pain, referred pain, and radicular pain. Pain. 2009;147(1-3):17-19.

12. Hines R, Keaney D, Moskowitz MH, Prakken S. Use of lidocaine patch 5\% for chronic low back pain: a report of four cases. Pain Med. 2002;3(4):361-365.

13. Nicolaou A, Nicholson B, Hans G, Brasseur L. Outcome predictors for treatment success with $5 \%$ lidocaine medicated plaster in low back pain with neuropathic components and neuropathic pain after surgical and nonsurgical trauma. J Pain Res. 2011;4:25-38.

14. Kern KU, Kohl M, Kiefer RT. Lidocaine patch for therapy of neuropathic and non-neuropathic pain. A clinical case series of 87 patients. Nervenarzt. 2010;81(12):1490-1497. German.
International Journal of General Medicine

\section{Publish your work in this journal}

The International Journal of General Medicine is an international, peer-reviewed open-access journal that focuses on general and internal medicine, pathogenesis, epidemiology, diagnosis, monitoring and treatment protocols. The journal is characterized by the rapid reporting of reviews, original research and clinical studies across all disease areas.

\section{Dovepress}

A key focus is the elucidation of disease processes and management protocols resulting in improved outcomes for the patient.The manuscript management system is completely online and includes a very quick and fair peer-review system. Visit http://www.dovepress.com/ testimonials.php to read real quotes from published authors. 